\title{
Reflections and analysis on the implementation of long-term nursing insurance for disabled athletes
}

\author{
Liu wei ${ }^{1, a}$, Yang Chujun ${ }^{2, b^{*}}$ \\ ${ }^{1}$ School of Physical Education, Harbin Business University ,Harbin, Heilongjiang, China \\ ${ }^{2}$ School of Finance and Public Administration, Harbin Business University, Harbin, Heilongjiang, China \\ a1394969630@qq.com \\ b*2959425079@qq.com
}

\begin{abstract}
In order to meet the real needs of the long-term survival and development of disabled athletes and the objective requirements of sports talent reserve, it is urgent to establish and improve the social insurance system for athletes. Based on the pilot status of athlete insurance and long-term nursing insurance in China, combined with the characteristics of athletes' industry, the imperfection of the disability protection system and the theoretical guidance of people-oriented, this theory is analyzed on the necessity of the implementation of long-term nursing insurance for disabled athletes, and provides theoretical ideas for their future development from the aspects of system construction, financing mechanism and professional training.
\end{abstract}

Keywords: Disabled athletes, Long-term care, Social insurance

\section{实施伤残运动员长期护理保险的思考与分析}

\author{
刘巍 $1, \mathrm{a}$ 杨楚军 $2, \mathrm{~b}^{*}$
}

${ }^{1}$ 哈尔滨商业大学体育学院，哈尔滨，黑龙江，中国

2 哈尔滨商业大学财政与公共管理学院, 哈尔滨, 黑龙江, 中国

a 1394969630@qq.com

b*2959425079@qq.com

\section{摘要}

为了满足伤残运动员长期生存发展的现实需要以及体育人才储备的客观要求, 建立健全运动员社会保险制度刻 不容缓。基于我国运动员保险及长期护理保险的试点现状，并结合运动员行业特性、伤残保障体系的不完善以 及以人为本的理论指导, 对伤残运动员长期护理保险实施的必要性进行分析, 并从制度建设、筹资机制以及专 业人才培养等方面为其未来发展提供理论思路。

关键词: 伤残运动员，长期护理，社会保险

\section{1. 前言}

随着东京奥运会和全运会的完美闭幕，不少优秀 运动员成为舆论讨论的热点, 无论是万众期待的奥运 常胜将军抑或是杀出重围的后起之秀, 荣耀背后免不 了伤病的伴随。我国著名女子体操运动员桑兰意外摔 伤、前国家男子排球运动员汤沝重伤痽疾以及武术散
打优秀运动员上官鹏飞不幸逝世等报道将一个个优 秀运动员的意外遭遇为大众知晓 ${ }^{[1]}$ 。人们难免将高成 就与高风险相联系起来, 不禁深思运动员为了夺取金 牌而付出的 “伤残代价” 是否值得以及如何通过运动 员社会保险制度来对优秀运动员戛然而止的运动生 涯、突如其来的收入损失以及未来极有可能陷入的生 活困境给予保障等问题。 
我国职业运动员人数不断扩大，据统计，2010 年在编与试训运动员总数高达 66588 人, 年均伤残率 达到 7.92\%，并呈现出运动员职业的高风险、高致残 率等特性。伤残运动员重残之后亟待解决的长期护理 与生活照料无疑是运动员及其家庭所面临的重要难 题之一。基于这一残酷现实，运动员长期护理保险的 实施是十分必要与值得的。

\section{2. 运动员长期护理保险发展现状分析}

我国运动员保险主要呈现行业保险与社会保险 相结合，商业保险予以补充的格局。相当一部分运动 员保险门槛较高, 只针对于高水平或获得大赛参赛权 的运动员, 加之运动员保险意识普遍不高, 体制内运 动员所享有的保障力度有限、体制外获得保障的更是 少之又少。此外, 基本医疗保障体系对于运动员的保 障力度十分有限, 运动员收入水平普遍不高, 基于收 入水平的待遇给付显然无法满足其需要。保障范围狭 窄, 仅在编运动员能够纳入城镇职工社保享受同等福 利待遇, 体制外运动员的保障权益被 “边缘化”。就 其保障深度与广度而言, 与竞技体育高危性、高重伤 率极其不匹配，未能体现行业特殊性。

当前我国长期护理保险在多地的试点推行已颇 具规模, 甚至有成为继医疗、养老、工伤、失业四大 险种之后第五大险的趋势。当前长护险的保障重点仍 集中于高龄失能老年人群, 对于失能失智及长期慢性 病群体缺乏关注。运动员与其他人群相比具有较高的 失能风险, 使得该群体对于长期护理的潜在需求较大。 运动员普遍职业寿命短、基本收入偏低、保险意识贵 乏, 即抵御风险的能力更弱。因而将运动员群体逐步 纳入到长期护理保险的保障范围是该险作为独立、新 型社会保险的一大发展趋势。

\section{3. 实施运动员长期护理保险的必要性分析}

\section{1. 我国运动员群体的行业特性}

高压训练下引发的高重伤率以及退役后职业病 的加剧性增大了运动员失能可能。我国竞技体育长期 处于举国体制模式, 政府包揽一切, 运动员、教练员 “唯金牌论” 思想严重。运动员在高强度训练下不断 突破自身极限, 运动时间集中且普遍较长, 训练准备 及恢复阶段不够, 再加上训练设施器材的防护性与规 范性不足等原因, 导致我国体育运动伤残风险总体呈 现出 “两低一高” 特征, 即低伤残率、低死亡率、高 重伤率。运动员疲劳训练, 带伤比赛屡见不鲜, 累积 性及突发性运动重伤成为高重伤率的主要成因 ${ }^{[2]}$ 。

此外, 运动员伤病存在隐形及潜伏性特征, 某些 疾病甚至延迟至退役后才显现。并随着运动员年龄的 增大, 伤病恶化迅速, 病情加剧急速。而退役后的运 动员往往面临再就业困境, 收入不稳定, 无法及时就 医治疗, 因病致贫, 因贫致残风险加剧。

\section{2. 运动员伤残保障体系的不完善}

现阶段我国运动员伤残保障体系主要包括国家 职工工伤保险、面向高水平运动员的优秀运动员伤残 互助保险以及市场化的人身意外伤害险等。主要保障 对象集中在省队、国家队的正式在编运动员以及从事 奥运会、全运会项目的专业运动员, 对于广大底层编 外运动员的保障存在大量空白。

作为运动员伤残保障体系核心的伤残互助保险, 其本质是体育系统内的伤残补偿, 而不合理的伤残等 级赔付标准却制约了伤残互助保险的发展 ${ }^{[2]}$ 。小额赔 付数量占比颇高, 对于轻度伤残运动员来说确实具有 一定成效，但最高赔付额仅 30 万元，对于重、特级 伤残运动员显得杯水车薪, 且一次性现金赔付仅考虑 到当期运动员伤病治疗费用补偿, 却忽视了未来更重 要、更长阶段的护理照料需要。这种重现金赔付的保 障给付手段对于那些护理需求远大于医疗需求的伤 残运动员来说是十分低效且不合理的。

\section{3. 以人为本理念的必然要求}

以人为本, 突破自我是体育事业的重要理念。运 动员是带动体育产业发展的关键力量, 运动员的切身 利益无法保障, 社会对于运动员的职业期待降低, 对 于运动员的荣誉淡漠处之, 必然不利于体育事业的可 持续发展。

近年来，国家加大了对于竞技体育的投资力度， 而运动员的人身保障也一直是竞技体育事业发展的 核心命题。早在建国初期, 贺龙就提出对于运动员“我 们要管一辈子”, 而运动员职业的高危性与高淘汰性、 伤残医疗保障体系的不完善又使得许多想要从事体 育事业的人们望而却步，对于竞技体育的人才储备十 分不利。当前许多大城市都面临招生困难, 体育人才 后备来源枯竭的危险 ${ }^{[3]}$ 。父母对于进入体育行业的前 景存有疑虑, 对于孩子未来生活发展存在担忧。因此 必然通过更完备的运动员保障体系, 令人满意的福利 待遇, 积极的医疗与养老就业保障政策, 来化解当前 竞技体育人才的流失与枯竭的严峻局面。

\section{4. 实施运动员长期护理保险的基本构想}

\section{1. 竭力填补运动员社会保障立法空缺}

政策法规所独有的约束力是制度有效实施的保 证。我国运动员社会保险与西方发达国家相比起步较 晚, 且尚未建立专门的法律。2002 年, 国家体育总 局颁布《优秀运动员伤残互助保险试行办法》, 明确 运动员个人主体责任及运动员伤残保险具体实施部 门; 2006 年国家体育总局出台《关于进一步加强运 动员社会保障工作的通知》(体人字 $\left(\begin{array}{llll}2 & 0 & 0 & 6\end{array}\right) 4$ 78 号) 指出要将编制内运动员逐步纳入到当地社会 保障覆盖范围并为运动员建立完善的社会保险基础 档案; 2010 年 4 月国家体育总局等 5 部门共同出台 
了《关于贯彻落实<关于进一步加强运动员文化教育 和运动员保障工作的指导意见>的通知》推进运动员 加入工伤保险工作，强化了运动员社会保障待遇 ${ }^{[1]}$ 。

不难看出在较长的一段时间里, 我国运动员社会 保险的政策推进是缓慢而渐进式的, 对于较高水平的 优秀运动员主要实行 “包下来” 的优待性、保护性政 策, 而对于体制外的普通运动员的保障政策则是少之 又少，前后两者有等级分化的趋势，与社会保障公平 性原则相违背。应当在充分考虑我国实际情况, 并借 鉴国外成熟运动员社保体系的基础上, 着力建成专属 于运动员保险的法律法规, 竭力将所有的运动员都纳 入到社会保险体系。重点解决当前运动员社会保障制 度中保障项目单一, 保障对象缺乏针对性, 政策覆盖 面窄以及与经济发展不相适应的 “无限政府” 责任意 识等问题。此外, 在未来更加市场化更加多元化的政 策大环境下, 责任体系主体的构建也应逐渐呈现出更 加多方的趋势, 因此立法过程中也需考虑实际情况, 动态调整政府、个人、社会三方责任比重。

\section{2. 借鉴试点地区经验，渐进式推进}

自 2016 年启动第一批长期护理保险制度试点以 来, 各试点地区积极探索、积累经验, 为之后全国范 围内的推行提供了卓有成效的示范作用。但不可否认 的事实是, 当前我国长期护理保险试点地区制度碎片 化和差异化问题突出, 必须采用渐进式推进方法, 在 借鉴试点地区经验基础上, 逐步扩大试点范围, 以图 建立覆盖全民的长期护理保险。

当前政策实施保障范围主要覆盖职工基本医疗 保险 (以下简称职工医保) 参保人群, 但一些具有地 方特色的制度设立也为运动员长期护理保险的加入 提供了不少的可能。例如成都市结合本市实际, 自主 研发创建了成都市长期失能照护等级评定标准, 为运 动员群体根据其行业特性与职业伤病规律建立具有 特色的失能照护等级评定标准提供思路。此外, 运动 员竞技项目各具特色, 失能风险存在差异, 如何合理 有效设置缴费比例, 保证效率的同时又不失公平, 是 需要探索与衡量的。而有些试点地区在筹资过程中注 重权利义务对等原则, 实行个人账户划转费率与失能 风险挂钩等举措, 为运动员长期护理保险的实施提供 了实践基础 ${ }^{[4]}$ 。

\section{3. 逐步建立互助共济、责任共担的筹资机 制}

筹资是长期护理保险的核心，也是构成保险资金 的重要基础 ${ }^{[5]}$ 。对于运动员来说, 资金缺口大与社保 统筹基金转移接续困难一直以来是运动员保险发展 的难点与障碍。

\section{3. 1 , 将运动员逐步纳入当地社会保障范围} 中

长期低收入的普通伤残运动员很难形成保险基 金积累, 加之日常医疗费用消耗大, 因而加快将运动 员纳入社会保障基金的统筹层次是解决基金统筹层 次低的关键举措。 2020 年 9 月, 中国医保局会同财 政部印发了《关于扩大长期护理保险制度试点的指导 意见》(医保发 (2020) 37 号)。文件指出, 参保对 象从职工基本医疗保险参保人群起步, 探索建立以互 助共济的方式筹资资金的社会保险制度 ${ }^{[6]}$ 。

在当前运动员保险碎片化的背景下，只有少部分 的优秀运动员被纳入到社会保障范围之内, 而长期护 理保险作为一种新型、独立的社会保险, 就应该将所 有符合保障要求的人群强制性的应保、尽保。除了现 役在编的优秀运动员, 还包括体制外的试训、在训运 动员以及高校大学生运动员。这样一个较大基数的运 动员群体的加入, 才能更有效地加快推动长护险的实 施。并通过大数法则原理, 加强社会共担风险能力, 对伤残运动员失能后的生活照料提供保障。与此同时, 还能够间接解决因地域流动性而导致的社保关系的 转移接续困难问题。

\section{3. 2 , 将运动员的个人缴费比例与失能风险 相挂钩}

运动员不同项目之间的伤残风险存在较高差异， 体操、滑雪、柔道摔跤等高难度或者高对抗性的项目, 其伤残几率以及致残率都远远高于一般项目, 例如射 击、羽毛球等。此外, 运动员的年龄也是其伤残严重 程度的又一影响因素, 年轻的运动员身体素质好, 治 愈能力和抗击打能力强, 而随着年龄增大, 其身体机 能下降, 外加累积性伤病的影响, 伤残风险加大。因 此在设定个人缴费比率时, 需要考虑到运动员职业特 性，即从事项目的高风险性、运动周期长短等。

此外，对于不同等级的运动员，其获得的保障能 力也是不同的。优秀运动员获得的奖项多, 社会影响 力大, 能够吸引商业保险公司为其投保, 保险意识以 及防范风险的能力实际上大于众多普通运动员。因此 通过将个人缴费比例与失能风险挂钩, 能很大程度化 解运动员因个体间的差异而造成的待遇不公平感，同 时在防止护理资源浪费，控制服务费用增速上也颇有 效用。

\section{3. 3 , 多渠道扩充运动员长护险的资金来源}

当前试点地区的长期护理保险基金对医疗保险 基金的依赖性较强, 大多采用一次性划拨方法将医保 基金的一部分移作长期护理保险的基金使用，长此以 往必然出现入不敷出的局面。此时若想实施运动员长 期护理保险，必然需要多渠道拓宽资金筹集方式，充 分发挥运动项目的市场性与商业性质, 带动体育产业 的发展, 吸引更多的商业保险的投资与补充 ${ }^{[7]}$ 。 
同时在国家、社会以及运动员三方责任共担的基 础上，开辟新的资金筹集渠道，用以填补运动员长护 险实施后所出现的巨大的基金缺口。例如，从大型的 商业性质的体育赛事的门票收入以及转播收益中提 取一定的比例用于运动员保险基金使用; 在每场体育 赛事的商业赞助中抽取一部分用于保险基金的积累。 大力发展全民性质的体育公益事业, 将一定的收入用 作运动员的保障 ${ }^{[1]}$ 。对于参加奥运会或者全运会的运 动员给予一定的奖励性补贴, 不仅针对于获奖选手, 对于所有获得参赛资格的运动员都应当给予一定的 补贴，可以通过补贴保险基金的方式获得。

\section{4. 专业服务机构建设与人才培养}

长期护理保险不同于一般的社会保险, 待遇给付 支付方式特殊, 且涉及到照护服务机构、照护保险服 务中心、辅具租赁公司等多个方面, 目前已经体现出 服务支付为主，现金支付为辅的特征。而对于伤残运 动员来说, 不仅需要专业的护理人员的服务, 还需要 懂得体育康复知识的专业人员的指导, 可以从运动队 队医队伍中挑选一部分人才, 经过系统培训后, 分批 次轮换扩充到运动员长期护理服务的人才队伍中, 对 护理服务人员的服务进行指导与监督。此外, 对于护 理学校应当增设体育康复专业, 用于运动康复方面的 护理人才储备。

\section{5. 结论}

我国长期护理保险目前仍处于起步阶段, 发展尚 不成熟。谨防制度差异化与碎片化带来的现实问题, 运动员这一特殊群体的加入无论是在制度设计、资金 安排上都需要进行多角度考虑, 需要更加合理有效、 操作性强的手段保证运动员长期护理保险与其他保 障体系的顺利衔接, 为建立健全运动员社会保障体系 增添力量。

\section{项目基金}

本文为 2020 年度黑龙江省社会科学研究项目 《后疫情时代冰雪体育消费稳增长研究》 (20429) 的 阶段性成果之一。

\section{References}

[1] Tian S., (2019) Study on the long-term care social insurance system for outstanding retired disabled athletes in China. Suzhou University.

[2] Ye MH, Wu XP., (2011)Research on the risk of sports disability and its prevention in China is based on the data of disability mutual insurance claims of outstanding athletes from 2007 to 2010 . Insurance research, (10):68-76.

[3] Sun Y, Chen ZL.,( 2015) Optimizing the path selection and implementation strategy of athlete insurance structure. Journal of Chengdu Institute of Physical Education, 41(01):62-68.

[4] Thompson, J.N. (1984) Insect Diversity and the Trophic Structure of Communities. In: Ecological Entomology. New York. pp. 165-178.

[5] Sun J., (2021)The experience, problems and policy suggestions of the long - term nursing insurance pilot in China. Price theory and practice, 1-6.

[6] Fu SJ, Zhang LW, Fang Y.,(2021) The research progress of long - term nursing insurance financing mechanism at home and abroad. Public health in China, 1-3.

[7] Tang W, Su F.,(2021) Study on different financing models of long - term nursing insurance. Financial research, 1-17. 